\title{
Semen Parameters And Pregnancy Rate Post Varicocelectomy For Subfertile Men With Sperm Count Less Than 10 Million, Sohag Medical School Experience. Ahmed Mahmoud Attia ${ }^{1}$, ELnisr Rashed Mohamed ${ }^{1}$, Mohamed Zaki Ali $^{2}$, Abdelbasset Abdou Mohamed Badawy ${ }^{1}$. Department of Urology, Faculty of Medicine, Sohag University
}

\begin{abstract}
Purpose: Despite Varicocele repair can improve fertility in infertile men with oligospermia, Conflicting opinions remain as to whether a varicocele repair improves fertility in individuals with severe oligospermia. The present study evaluated the benefits of varicocelectomy in men with severe oligospermia and clinically palpable varicocele in terms of the spontaneous pregnancy rate and improvement in semen analysis parameters. Materials and Methods: This was a prospective study of 50 men with oligospermia who underwent varicocelectomy at our center in the period between April 2017 to August 2018. The subjects were followed for a mean period of 3 months. The outcome was compared among groups of men with moderate $(5-10$ million $/ \mathrm{mL})$, and severe $(<5$ million $/ \mathrm{mL}$ ) oligospermia who were operated on during the same period. Results: A total of 11 of 32 men $(34.37 \%)$ with moderate OS achieved spontaneous pregnancy. Mean sperm density increased from 7.36 million/mL preoperatively to 27.5 million/mL postoperatively. The mean time to pregnancy from the date of surgery was 3 months. The spontaneous pregnancy rate in men with severe OS was $22.2 \%$. Conclusion: This study recommends varicocelectomy in patients with severe oligospermia as there was significant improvement in semen parameters and pregnancy rate and thus provides a chance for such patients to achieve unassisted pregnancy before trying assisted reproductive techniques.

Keywords:Varicocele, severe oligosp-ermia, preg-nancy rate

\section{INTRODUCTION:}

Varicocele is an abnormally dilated veins in the pampiniform plexus of the spermatic cord (10). Infertility is found in $10-15 \%$ of couples, and men are found to be responsible for infertility in $40 \%$ of cases $(\mathbf{1})$. Varicocele is the major reversible cause of infertility in males (4). several theories have been proposed to contribute to varicocele induced infertility include increased oxidative stress, local hypoxia, scrotal

hyperthermia, hormonal disturbances, autoimmunity, testicular venous hypertension, and increase in spermatic vein catecholamines $(\mathbf{8})$. There are various surgical approaches for varicocelectomy. These include laparoscopic varicocelectomy, microsurgical varicocelectomy, and open varicocelectomy. To assess the effect of varicocele repair in men with oligospermia and palpable varicocele as
\end{abstract}


regard pregnancy rate and improvement in semen analysis variables (sperm morphology, sperm motility, and sperm concentration). We conducted this study on 50 patients with sperm count less than 10 million $/ \mathrm{mL}$.

MATERIALS AND METHODS: This study was conducted in the andrology unit, urology department, Sohag faculty of medicine in the period between April 2017 to August 2018 and 54 infertile patients with clinical varicocele and oligospermia (sperm count less than 10 million $/ \mathrm{ml}$ ) enrolled in this study, but only 50 patient who was completed follow up in the andrology unit and the other 4 patients lost in follow up. After explanation of the procedure, possibility of the complications and results, and informed written consent from all patients was obtained. All Patients subjected to meticulous and careful physical examination, scrotal Duplex, semen analysis (at least two semen analyses performed before and two semen analyses performed after surgery) and routine laboratory investigations. We included Patients with Sperm count is less than 10 million $/ \mathrm{ml}$, normal hormonal profile (FSH, LH, Prolactin, and Testosterone), normal karyotyping, and Age of the wife is less than 40 years. We excluded patients with testicular atrophy, azoospermia, and recurrent varicocele. All patients are subjected to microsurgical sub inguinal varicocelectomy. The position of the external inguinal ring is identified then small incision about $2.5 \mathrm{~cm}$ is done directly below the external ring. The spermatic cord is elevated gently into the field followed by opening of the external spermatic fascia, the cremasteric muscle and fascia, and the internal spermatic fascia, and the testicular artery, lymphatics, and vas deferens are identified and preserved then identification and ligation of dilated veins. Semen analysis was obtained three months after varicocele repair. All patient was informed about the procedure, possible complications and results. Approval from ethical committee of sohag faculty of medicine was obtained.

Statistical analysis: Data was analyzed using STATA version 14. 2 (Stata Statistical Software: Release 14.2 College Station, TX: StataCorp LP.). Quantitative data were represented as mean, standard deviation, median, and range. Data were analyzed using student t-test. When the data were not normally distributed Mann-Whitney test was used. Qualitative data were presented as number and percentage and compared using either Chi-square test or Fisher exact test. Pre and post semen parameters were compared using Wilcoxon matched paired test. Spearman correlation analysis was done to find correlation between duration of infertility and semen parameters.

Graphs were produced by using Excel or STATA program. P-value was considered significant if it was less than 0.05 .

RESULTS: This study included 50 patients with a mean age of 30.82 years. 16 patients were cigarette smokers and 20 patients were shisha smokers. The median duration of marriage was 4 years. 2 patients had a history of previous marriage. The median age of the wives was 27 years. The menstrual cycles were regular with no demonstrable infertility factor. In the current study 44 patients out of 50 were presented with primary infertility while 6 patients have 2ry infertility and two of them had offsprings (the youngest was 3 years). The median infertility duration 
was 3 years. No patient had erectile dysfunction while 5 patients have a history of premature ejaculation and 7 patients have a history of irregular marital life due to traveling abroad. We noticed that 1 patient had a history of right congenital hernia repair, also 1 patient had a history of burn to the lower abdomen but external genitalia was not affected. In our study one patient had a history of previous testicular biopsy which was positive and did cryopreservation. 4 patients had a history of ART but all had a history of failure. In the present study, one patient was diabetic on insulin, and also 1 patient had a history of poliomyelitis and one patient was hepatitis B virus-positive. Two patients had a family history of infertility but only one patient had a family history of varicocele. General examination of the patients revealed that the median BMI was 24, well developed secondary sexual characters, absence of signs of hypogonadism and signs of hyperprolactinemia. 1 patient had inguinal scar of previous hernia repair, 1 patient had appendectomy Scar, 1 patient had burn Sacr, 1 patient had Bilateral varicose veins, and 1 patient had left lower-limb paralysis(previous poliomyelitis). Local examination of the patients revealed that one patient with reduced testicular size and another patient with epididymal cyst (small). In our study, we noticed that there were 85 testicles with varicocele(13 grade one, 44 grade two and 28 grade three varicocele). All patient did full hormonal assay, the mean serum FSH level was $5.32 \mathrm{~m} \mathrm{IU} / \mathrm{ml}$, the mean serum $\mathrm{LH}$ level was $5.41 \mathrm{~m} \mathrm{IU} / \mathrm{ml}$, the mean serum total testosterone level was 6.40 $\mathrm{ng} / \mathrm{ml}$, the mean serum free testosterone level was $17.99 \mathrm{pg} / \mathrm{ml}$, the mean serum estradiol level was $20.11 \mathrm{pg} / \mathrm{ml}$, and the mean serum PRL level was $9.65 \mathrm{ng} / \mathrm{ml}$. Also, all patients did chromosomal study which revealed that all patients were 46 $x y$. In our study, all patient did a preoperative duplex ultrasound that revealed that all patient with normal testicular size except one patient with reduced testicular size, only one patient with left epididymal cyst, 35 patient with bilateral varicocele, 13 patient have varicocele on left side and 2 patients have varicocele on right side. only one patient did TRUS which revealed no obstruction at all. All patients subjected to sub inguinal varicocelectomy. In our study, pregnancy rate for patients with sperm count less than 5 million $/ \mathrm{ml}$ was $22.2 \%$, while for patients with sperm count more than 5 million $/ \mathrm{ml}$ was $34.37 \%$ ). We did postoperative scrotal ultrasound to assess the change in testicular size, we noticed that no significant change in the size of the testis. An increased period of infertility was associated with negative impact on semen variables. There were statistically significant changes in morphology, progressive motility, and total motility ( $\mathrm{p}$ $<.005, \mathrm{p}<0.01$, and $\mathrm{p}<0.0001$ respectively ).

DISCUSSION: In our study of 50 infertile patients with clinical varicocele and oligospermia, Patients were categorized into two categories with moderate and severe oligospermia. In the moderate oligospermia category, the mean post-operative sperm concentration improved from $7.36 \pm 1.52 \mathrm{million} / \mathrm{mL}$ to $27.5 \pm 12.06$ million $/ \mathrm{mL}$ ( $\mathrm{p}$-value $<0.001)$. In the severe oligospermia category, the mean post operative sperm concentration improved from $2.86 \pm 1.46$ million $/ \mathrm{mL}$ to $13.7 \pm 6.8 \mathrm{million} / \mathrm{mL}$ (pvalue $<0.001)$. Our study showed natural pregnancy rates of $34.37 \%$, and $22.2 \%$ in the moderate, and severe oligospermia 
categories, respectively. Enatsu et al., 2014, evaluated infertile men with severe oligoasthenozoospermia, the mean sperm count increased from 2.4 to $11.6 \mathrm{million} / \mathrm{mL}(\mathrm{p}<0.001)$, but natural pregnancy rate was $16.7 \%$ (5). In addition, Gupta et al., 2018, retrospectively evaluated infertile men with varicocele and oligozoospermia and noticed that in moderate oligospermia category, the mean sperm count improved from $7.10 \mathrm{million} / \mathrm{mL}$ to 19.38 million $/ \mathrm{mL}(p-$ value $=0.049)$, while in the severe oligospermia category, the mean sperm count was improved from $2.29 \mathrm{million} / \mathrm{mL}$ to $14.09 \mathrm{million} / \mathrm{mL}$ ( $\mathrm{p}$ value $=0.026$ ) and the natural pregnancy rates were $46.2 \%$ and $37.1 \%$ in the moderate and severe oligospermia categories $(\mathbf{6})$. The mean duration of follow up was 12.4 months, while in our study, the duration of follow up was 3 months only. In the present study, we noticed that there was significant improvement in the progressive and total motility postoperatively (from $19.52 \%$ to $34.77 \%$ and from $36.99 \%$ to $53.74 \%$, respectively, both $\mathrm{P}<0.0001)$. Baazeem, et al., 2011, noticed significant increases in total motility ( $10.86 \%, \mathrm{P}<0.0001)$ and in progressive motility ( $9.69 \%, P=0.003)(2)$. Also, Enatsu et al., 2014, reported that varicocelectomy for men with severe oligospermia was associated with significant increases in mean sperm motility (from $32.8 \%$ to $42.2 \%$, $\mathrm{p}=0.0024)(5)$. In addition, Shamsa et al., 2010, reported that there were significant increases in sperm motility (from $38.3 \%$ to $41.1 \%, \mathrm{P}<0.01$ ) after varicocele repair (12). In our study, sperm morphology was improved significantly after varicocele repair (from $43.2 \%$ to $49.64 \%, P=0.03$ ). Shamsa et al., 2010, reported that sperm morphology improved significantly after varicocele repair (from $54.5 \%$ to $56.5 \%$, $\mathrm{P}<0.01)$ (11). Also, Gupta et al., 2018, noticed that varicocelectomy for patients with severe oligospermia followed by improvement of sperm morphology (from $39.91 \%$ to $44.74 \%)(6)$. There is ongoing debate about the effect of bilateral vs unilateral varicocele repair on the outcome of surgery. The present study showed that sperm motility was improved significantly after bilateral varicocelectomy $(\mathrm{P}<.05)$ in men with bilateral varicoceles which go with the results of Libman et al., 2006, who reported that men who subjected to bilateral varicocelectomy had a statistically significant increases in sperm motility than men who did unilateral varicocelectomy (7). we noticed that the sperm parameters correlate negatively with the increased period of infertility. The change in progressive motility, total motility and morphology were statistically significant $(\mathrm{p}<0.01, \mathrm{p}<0.0001$ and $\mathrm{p}<.005$ respectively), which go with results of Chehval and Purcell, 1992, who demonstrated a decline in sperm parameters over time in men with untreated varicocele (3). Also, Zorba et al., 2009, found that after varicocelectomy, the results of semen parameters correlated negatively with the duration of infertility which is in agreement with this $\operatorname{study}(\mathbf{1 2})$. multivariate analysis, including age of wife, varicocele grade, body mass index, unilateral versus bilateral varicocelectomy and serum prolactin level which were statistically significant in univariate analysis $(\mathrm{P}<.05)$. We found that the age of wife was the only independent factor affecting pregnancy rate, which is in agreement with 
Nieschlag et al., 1998, who conducted a randomized controlled trials reported that the wives achieving a pregnancy were significantly younger than those wives not achieving a pregnancy (28.8 years versus 31.2 years, $P<0.05)(9)$.

CONCLUSIONS: This study recommends varicocele repair in patients with severe oligospermia as there was significant improvement in semen analysis variables and pregnancy rate. Especially patients with sperm concentration $>5$ millionl $/ \mathrm{ml}$, who have a significant improvement in pregnancy rate and semen parameters (pregnancy rate was $34.37 \%$, the mean postoperative sperm concentration was improved from 7.36 to 27.5 million $/ \mathrm{mL}$, p-value $<0.001$ ), while in patients with sperm concentration $<5$ millionl $/ \mathrm{ml}$ (pregnancy rate was $22.2 \%$, the mean post-operative sperm concentration was improved from 2.86 to $13.7 \mathrm{million} / \mathrm{mL}$, p-value <0.001). Our study recommends bilateral varicocelectomy in patient with bilateral varicocele which has impact on post-operative sperm motility, also recommends ICSI in longer duration of infertility and older age of the wife.

\section{CONFLICTS OF INTEREST:}

The authors have nothing to disclose.

\section{REFERENCES:}

1- Agarwal A, Mulgund A, Hamada A, Chyatte MR. A unique view on male infertility around the globe. Reprod Biol Endocrinol. 2015; 13: 37.

2- Baazeem A. et al., Varicocele and male factor infertility treatment: a new metaanalysis and review of the role of varicocele repair. Eur. Urol. 2011; 60, 796-808.

3- Chehval MJ, Purcell MH: Deterioration of semen parameters over time in men with untreated varicocele:evidence of progressive testicular damage. FertilSteril. 1992; 57: 174-7.

4- Dubin L. \& Amelar R D. Etiologic factors in 1294 consecutive cases of male infertility. Fertil Steril. 1971;22, 469-74.

5- Enatsu N, Yamaguchi K, Chiba K, Miyake H, Fujisawa M. Clinical Outcome of Microsurgical Varicocelectomy in Infertile Men With Severe Oligozoospermia. Urology. 2014;83(5):1071-1074

6- Gupta C, Chinchole A, Shah R, Pathak H, Talreja D, Kayal A. Microscopic varicocelectomy as a treatment option for patients with severe oligospermia. Investigative and clinical urology. 2018;59(3):182-186.

7- Libman J, Jarvi K, Lo K, Zini A. Beneficial effect of microsurgical varicocelectomy is superior for men with bilateral versus unilateral repair. J Urol 2006; 176: 2602-5

8- Marmar J L. The pathophysiology of varicoceles in the light of current molecular and genetic information. Hum Reprod Update. 2001; 7, 461-72.

9- Nieschlag E., Hertle L., Fischedick A., Abshagen K. \& Behre H M. Update on treatment of varicocele: counselling as effective as occlusion of the vena spermatica. Hum Reprod. 1998; 13, 2147-50.

10- Owen R C., Mccormick B J., Figler B D. \& Coward R M. A review of varicocele repair for pain. Transl Androl Urol. 2017; 6, S20-s29.

11- Shamsa A, Nademi M, Aqaee M, Fard AN, Molaei M. Complications and the effect of varicocelectomy on semen analysis, fertility, early ejaculation and spontaneous abortion. Saudi J Kidney Dis Transpl 2010; 21: 1100-5.

12- Zorba UO, Sanli O M, Tezer M, Erdemir F, Shavakhabov S, Kadioglu A: Effect of infertility duration on post varicocelectomy sperm counts and pregnancy rates. Urology. 2009; 73: 76771. 